\title{
As the cube turns: Evidence for two processes in the perception of a dynamic reversible figure
}

\author{
GERALD M. LONG, THOMAS C. TOPPINO, and JOHN F. KOSTENBAUDER \\ Villanova University, Villanova, Pennsylvania
}

\begin{abstract}
The reported reversals of a rotating Necker cube, which changes direction of rotation when a perspective reversal occurs, were examined under a number of conditions. These permitted comparisons of reversal rates within viewing periods, across successive viewing periods within an experimental session, and across successive weekly sessions. In addition, observers viewed either one or two rotating cubes simultaneously within each of the various viewing periods. Clear evidence for a learning effect was obtained in the form of significant savings across successive viewing periods and sessions. At the same time, results from the multiple-cube conditions and from the pattern of reversals within individual viewing periods appeared to be more consistent with a process of neural fatigue. A two-stage model of reversible-figure perception is proposed which is characterized by (1) fatiguing with extended viewing of the two sets of neural channels that underlie the two percepts of the reversible figure, and (2) learning, which helps to establish the organization of the cortical channels as well as moderate channel activity via such processes as attention and strategy.
\end{abstract}

In the last decade there has been a resurgence of theoretical and empirical activity related to a particular class of visual illusions most commonly referred to as reversible figures. This class of illusions includes such well-known figures as the Necker cube, Rubin's vase-faces, the Schroeder staircase, the Mach folded card, Fisher's man-girl, Boring's young girl-old woman, the Maltese cross, and several others (see Attneave, 1971, for examples of these and other reversible figures). Although empirical research with these figures can be traced back to the last century, there is still extensive interest in them because of the insights they are thought to provide into the nature of the perceptual process by their curious multistable character.

Two competing classes of theories have evolved in an attempt to explain the perceptual fluctuations reported for the Necker cube and other reversiblefigure illusions. ${ }^{1}$ The older theory, that of neural satiation (cf. Attneave, 1971; Howard, 1961; Kohler \& Wallach, 1944; Orbach, Ehrlich, \& Heath, 1963), proposes a passive process of fatigue as the basis for perceived reversals. In this view, the cortical organization underlying one perspective of a reversible figure satiates or fatigues with extended viewing. It then gives way to a second, fresher cortical organization underlying the other perspective. This

This article is adapted from the third author's Master's thesis at Villanova University. We wish to express our appreciation to Douglas Klieger for his helpful comments on various aspects of the research. Requests for reprints should be sent to the first author, Department of Psychology, Villanova University, Villanova, Pennsylvania 19085. cortical organization eventually tires also, and the original cortical organization, which has had an opportunity to recover while the second organization is dominant, once again takes over. However, because this cortical structure has not yet fully recovered from its fatigue, it now tires more quickly. Over an extended period of viewing, the structures alternate more frequently as they fatigue more rapidly, until some relatively constant rate of fatigue and recovery is achieved. The pattern of perceived reversals over time appears as a negatively accelerated function, which has been reported by numerous investigations with several reversible figures (e.g., Babich \& Standing, 1981; Brown, 1955; Cohen, 1959; Howard, 1961; Price, 1969a, 1969b; Spitz \& Lipman, 1962).

Initially, this satiation model was couched in terms of Gestalt physiology such that extended viewing resulted in an increase in the resistance to the flow of electric current in the brain tissue mediating the contours of the pattern (e.g., Kohler, 1960). This view was soon replaced, however, by a somewhat similar model in which satiation was conceptualized as "some kind of fatigue process of aggregates of neurons signaled by an augmentation of the photic response" (Orbach et al., 1963, p. 457). Most recently, investigators have attempted to relate the satiation model to fatiguing of the neural "channels" that are believed to comprise the visual system (cf. Regan, 1982). Numerous physiological and psychophysical studies have suggested that there are neural channels attuned to various stimulus characteristics such as spatial frequency (e.g., Blakemore \& Sutton, 1969; Campbell \& Robson, 1968), orientation (e.g., 
Blakemore \& Campbell, 1969; Blakemore \& Nachmias, 1971), motion (e.g., Beverley \& Regan, 1973; Levinson \& Sekuler, 1980), changing size (e.g., Petersik, Beverley, \& Regan, 1981; Regan \& Beverley, 1978; Regan \& Cynader, 1979), motion in depth (Chase \& Smith, 1981; Regan \& Beverley, 1979), disparity (Schumer \& Ganz, 1979), and possibly others (see Braddick, Campbell, \& Atkinson, 1978; Maffei, 1978; Regan, 1982). Moreover, in extensive research determining the effects of selectively adapting these proposed channels, it has been found that these channels appear to fatigue over time with extended viewing and to recover with sufficient rest-very similar to the behavior of the cortical organizations hypothesized to underlie reversible figures. It is not surprising, then, that Ginsberg (Note 1) and Palmer and Bucher (1981) have explicitly related the alternating percept of one type of reversible figures (ambiguous triangles) to the fatiguing of separate orientation channels in the visual system.

The other current interpretation of reversible figures has its basis within the information-processing framework of cognitive psychology, although precursors to this interpretation can be traced back at least as far as Ammons (1954; Ammons, Ulrich, \& Ammons, 1959). This interpretation depends on two logically separable processes. First, the perceptual alternations are attributed to a "cyclic decision process" (Vickers, 1972) or a "process of continuous trial and error on the part of the perceptual system" (Rock, 1975, p. 289). Hence, rather than reflecting the activity of spontaneous, generally passive brain processes, the perceptual reversals are conceptualized as alternating solutions to the perceptual puzzle provided by the stimulus. That is, in its attempt to attain the plausible solution or likely "hypothesis" to the problem of "what is there," the perceptual system is faced with two acceptable solutions and consequently alternates between the two. Second, the finding that reversal rate increases as a function of viewing time or experience is accounted for by a learning process. In this regard, the frequently reported asymptotic curve depicting number of reversals as a function of viewing time is thought to reflect a standard learning curve for the perceptual response involved.

Several findings have been cited as support for an interpretation of reversible figures in terms of active decisional processes and/or learning processes. These include the findings that observers have some volitional control over reversal rate (e.g., Pelton \& Solley, 1968), that an observer's prior knowledge of the reversible character of the stimulus affects reported reversals (Girgus, Rock, \& Egatz, 1977), and that significant practice effects across sessions have been reported (e.g., Ammons, 1954; Mefferd, Wieland, Greenstein, \& Leppman, 1968).

The present research was undertaken in an attempt to provide further insight into the processes underlying the perception of reversible figures. It was an- ticipated that the most plausible model would require an incorporation of both a passive fatigue process and an experiential or learning factor. To this end, a design was chosen that would be sensitive to the roles of both neural fatigue and learning in the reported reversals of a standard reversible figure. It was thought that the "either-or" nature of most prior research had tended to overlook the likely role of both processes in the phenomenon under investigation. Furthermore, the present research also employed a form of stimulus presentation suggested by Long and Toppino (1981) that was believed to provide unique insight into the processes underlying reversals. Specifically, this involved the simultaneous presentation of two identical reversible stimuli. As Long and Toppino have argued, the fatigue and the learning (or decisional) models would appear to make different predictions for the reversal rates obtained with these multiple figures. At the very least, it was hoped that the present investigations would provide empirically determined limits or constraints to the processes hypothesized to underlie the fluctuating character of reversible figures.

\section{Rationale}

The major postulate of current satiation theory is that neural fatigue underlies the perception of reversible figures and that this passive process is demonstrated by a negatively accelerated rate of reversals over time. In the present work, it was hypothesized that such a reversal rate would take place over $2 \mathrm{~min}$ of continuous viewing of a rotating Necker cube. This was based on numerous previous studies with stationary reversible figures (e.g., Babich \& Standing, 1981). More importantly, it was predicted that this identical pattern would repeat itself over the course of successive 2-min blocks of viewing, provided adequate rest between blocks was given to dissipate any neural fatigue that had built up. Furthermore, if a passive process of transient neural fatigue is the dominant process underlying the illusion, then there should be no difference in total reversals reported over the six 2-min blocks in a single session. However, if long-lasting adaptation (fatigue) is the basis for the effect, analogous to what several researchers have reported for various visual aftereffects (e.g., Favreau, 1979; Frome, Harris, \& Levinson, 1975; Hansel \& Mahmud, 1978), then a complete return to baseline from one viewing period to the next within a session is not predicted; but a return to baseline from one weekly session to the next would be expected. Hence, within a fatigue model, it is predicted that a nearly identical pattern of reversals will occur either from each 2-min block to another or from each weekly session to the next, depending upon the duration of the fatigue established by the adaptation.

Consider, however, the predicted pattern of results if learning is a dominant process underlying perceptual reversals. First, there would be an increase in 
reversal rate within each 2 -min block as well as a general increase in reversals from the first to the last of six 2-min blocks in a single viewing session. The learning/decisional model would expect experience and practice to increase in a manner reflective of a classic learning curve-and perceived reversals to increase accordingly throughout an experimental session. Similarly, if learning is, indeed, a dominant process underlying perceived reversals, long-term learning would also be evidenced. Hence, a significant increase in reversals would be expected over the course of four weekly sessions. It is unlikely that such a demonstration could result from a fatigue process, unless one is willing to propose an extremely robust fatigue of the cortical organizations. Rather, it is suggested that evidence of a progressive increment in the number of perceived reversals from successive sessions occurring at weekly intervals would most likely reveal the influence of practice and familiarity on the perceptual task involved.

Several additional comparisons involving the type of viewing conditions in this experiment were also planned to test the implications of Long and Toppino's (1981) claim that an observer is able to perceive reversals independently in each of two reversible figures at the same time. In the research to follow, while some observers were required to report the reversals of a single Necker cube, other observers were required to report the reversals of two cubes presented simultaneously in a side-by-side arrangement. In one of the viewing conditions (to be referred to as "double left"-DL), subjects counted reversals for only the cube on the left of fixation. In another ("double right"-DR), subjects counted reversals for only the cube on the right of fixation. In a third condition ("double both"-DB), subjects counted reversals for both cubes. It was hypothesized that, if the strict satiation model were accurate, the mean number of reversals reported in the $\mathrm{DL}$ condition would be equal to the mean number of reversals reported for the left cube only in the DB condition, and the mean number of reversals in the DR condition would be equal to the mean number of reversals reported for the right cube only in the DB condition. Because current satiation theory states that separate and fatigable channels underlie each perspective of a reversible figure, it would predict one set of channels to underlie the perceived reversals of a Necker cube in the right visual field and a separate set of channels to underlie the perceived reversals of the Necker cube in the left visual field. If these channels are assumed to have similar fatigue and recovery characteristics, the same number of reversals should result within each set of channels, regardless of the activity of the other set of channels. By comparing the number of reversals reported in the DL and DR conditions with those reported in the DB condition, this hypothesis could be tested directly.
On the other hand, postulating that reversals reflect the operation of an active decisional process leads to a different prediction. If two simultaneously presented cubes appear to reverse separately from one another as reported by Long and Toppino (1981), each reversal of each cube must involve a separate decision. Thus, fewer reversals would be perceived with each cube in the DB condition than for the single cube counted in the DL or DR conditions. This follows from the assumptions that the decision-making process is serial in nature and can complete only a certain number of decisions per unit time (e.g., Vickers, 1972). ${ }^{2}$ The decision-making time is divided between the two cubes in DB, whereas no such constraint on processing occurs in DL or DR, in which the observer attends to only a single cube. Hence, fewer decisions per cube would be made while counting the two cubes in DB than while counting the single cube in either DL or DR.

\section{METHOD}

\section{Subjects}

Fifty female and 50 male right-handed college students at Villanova University participated in this experiment. Only righthanded individuals were recruited and permitted to participate in this study. All subjects participated for partial fulfillment of the requirements of a general psychology course.

\section{Apparatus}

Subjects were seated $1 \mathrm{~m}$ away from a $125 \times 265 \mathrm{~mm}$ screen upon which shadows of a Necker cube, $45 \mathrm{~mm}$ on a side, were cast. The screen consisted of a translucent pane of glass covered by a piece of medium-weight white paper that served as a rearprojection system. Either one or two shadows of the Necker cube could be cast upon the screen by using either one or two $150-\mathrm{W}$ light bulbs positioned $95 \mathrm{~cm}$ apart and $88 \mathrm{~cm}$ from the Necker cube. The luminance on the white screen was $1.0 \mathrm{fL}$. The size of the shadow(s) of the cube on the display was $47 \mathrm{~mm}$ per side when a full front face was displayed. At the viewing distance of $1 \mathrm{~m}$, this corresponds to a visual angle of about $2.7 \mathrm{deg}$. The center of the cube(s) was $42.5 \mathrm{~mm}$ to the right and/or left of a $10-\mathrm{mm}$ black fixation point on the display. The cube rotated at a constant speed of 10 rotations/min. The subjects placed their index fingers on standard telegraph keys and reported each perceived reversal of direction of rotation of the cube(s) by pushing the left telegraph key if they were to report reversals of the left cube and/or pushing the right telegraph key if they were to report reversals of the right cube. Foringer equipment, including electronic counters, was connected to the telegraph keys and recorded each depression of the telegraph key corresporiding to a perceived reversal.

\section{Vlewing Conditions}

Twenty subjects (10 females and 10 males) were assigned randomly to each of three experimental viewing conditions:

Double Cube Left (DL). Subjects observed two cubes on either side of a central fixation point, but reported reversals for the left cube only by depressing a single telegraph key.

Double Cube Right (DR). Subjects observed two cubes on either side of the central fixation point but reported reversals for the right cube only by depressing a single telegraph key.

Double Cube Both (DB). Subjects observed and reported reversals of each of two cubes on either side of the central fixation point by depressing the left telegraph key when the left cube appeared to reverse and the right telegraph key when the right cube 
appeared to reverse. Extensive pilot work had indicated that subjects have no apparent difficulty with these instructions.

In addition to the above three groups, two further groups were included as control groups. The rationale for these groups will be treated in detail in the Discussion section. Twenty subjects (10 females and 10 males) were assigned randomly to each of these control conditions:

Single Cube Left (SL). Subjects observed a single cube placed to the left of a central fixation point and reported perceived reversals of rotation by depressing a single telegraph key each time the cube appeared to change in direction of rotation.

Single Cube Right (SR). Subjects observed a single cube placed to the right of the central fixation point and reported perceived reversals by depressing a single telegraph key.

And finally, three females and three males from each of the five conditions just described returned for three more identical experimental sessions at weekly intervals.

\section{Procedure}

After being randomly assigned to one of the five viewing conditions, each subject was given a 1 -min observation period to adapt to the apparatus and become familiar with reversals of both a stationary and a rotating Necker cube. After experiencing reversals, the subject was given a 2-min rest to allow for potential satiation effects from the initial viewing period to dissipate. The subject was then asked to observe the display passively and report, by depressing the appropriate telegraph $k e y(s)$, each reversal that occurred in a 2-min span. The experimenter recorded the number of reversals reported within each of the four $30-\mathrm{sec}$ intervals comprising the 2-min block. In the actual 30-min experimental session that followed, the subject viewed the rotating cube(s) in six 2 -min blocks with 2 -min rest periods between each. These values were chosen on the basis of pilot work that had indicated relatively little difficulty for observers while avoiding the general boredom or fatigue of longer viewing periods which often result in highly variable, "noisy" data (see Vickers, 1972). The subjects were urged to keep their eyes on the fixation point during all viewing periods. No independent check on fixation was made.

\section{RESULTS}

The conditions of interest in the present work involved the effect on perceived reversals of instructional conditions (DL, DR, and DB), the effect of successive 30 -sec intervals within each 2 -min viewing block, and the effect of successive 2-min blocks within the six blocks of an experimental session. In addition, the effect of successive experimental sessions was assessed for those observers run in four weekly sessions.

Figure 1 presents the mean number of reversals for the three instructional conditions as a function of 30-sec intervals collapsed across the six 2-min blocks. Figure 2 presents the mean number of reversals for the three instructional conditions as a function of the six 2-min blocks collapsed across the four 30-sec intervals. The significance of these patterns was assessed by a 3 (DL, DR, DB conditions) $\times 4$ (30-sec intervals) $\times 6$ (two-min blocks) ANOVA. The main effect of instructional conditions, evident in both figures, was highly significant $[F(2,57)=55.66, p<$ $.001]$. Probing this main effect with a Tukey a test revealed that significantly more reversals were reported in the DB condition than in either the DL

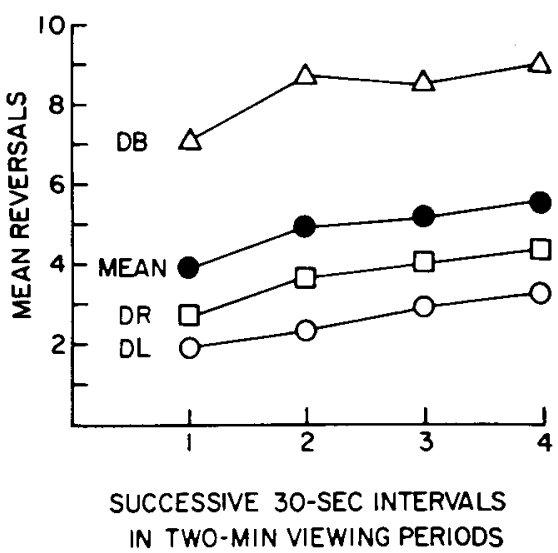

Figure 1. Mean reversals of the rotating Necker cube(s) reported in the three viewing conditions as a function of the 30 -sec intervals comprising the $2-\mathrm{min}$ viewing periods. The curve labeled "MEAN" presents the data collapsed across the three viewing conditions.

$(p<.01)$ or the DR condition $(p<.01)$. The reversals in the DL and DR conditions did not differ significantly from each other $(p>.05)$. In addition, to test predictions outlined in the introduction, the number of reversals reported in the DB condition were subdivided into those reported for the left cube, DB (left), and those reported for the right cube, DB (right). These were then compared with the number of reversals reported in the DL and DR conditions, respectively. The mean number of reversals in the DL condition (2.59) and the DB (left) condition (3.29) did not differ significantly $[\mathrm{t}(38)=1.76, \mathrm{p}>.05]$, but there were slightly more reversals in the DB (right) condition (5.04) than in the DR condition (3.71) [t(38) $=2.45, \mathrm{p}<.05$ ].

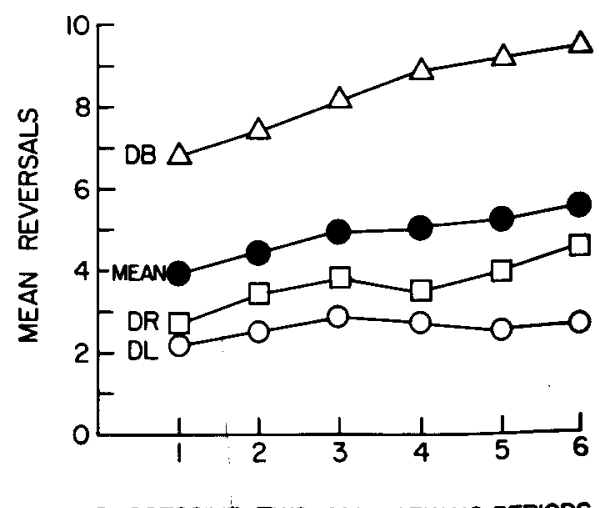

SUCCESSIVE TWO-MIN VIEWING PERIOOS

Figure 2. Mean reversals of the rotating Necker cubed(s) reported in the three viewing conditions as a function of the six 2-min viewing periods comprising the experimental seavion. The curve labeled "MEAN" presents the data collapsed acros the three viewing conditions. 
The main effect of intervals, shown in Figure 1 as a consistent increase in reversals over the four successive 30-sec intervals, was also highly significant $[F(3,171)=38.46, p<.001]$. The two-way interaction of conditions $\times$ intervals was not significant $[F(6,171)=1.70, p>.05]$. The differences across the four intervals were probed with a Tukey a test which revealed that the mean number of reversals in each of the four 30-sec intervals differed significantly from one another $(p<.05)$, except for the second and third interval, which did not differ significantly $(p>.05)$. A trend analysis revealed primarily a linear increase over successive intervals $[F(1,171)=105.39, p<.001]$, but with a small quadratic component $[F(1,171)=6.63, p<.05]$.

The main effect of blocks, shown in Figure 2 as an increase in reversals over the six 2-min blocks, was also significant $[F(5,285)=10.47, p<.01]$. However, the two-way interaction of conditions $x$ blocks was significant $[F(10,285)=2.79, p<.05]$. This is evident in Figure 2 from the much steeper rise for the DB condition than for either of the other two viewing conditions.

The three-way interaction of conditions $x$ intervals $\times$ blocks, although very small, was statistically significant $[F(30,855)=1.85, p<.01]$. Probing this interaction revealed that, for the DB condition only, there was a significant intervals $\times$ blocks interaction $[F(15,855)=2.55, p<.05]$. However, because of the size of this three-way interaction, which accounts for less than $1 \%$ of the total variance (Winer, 1971, pp. 428-430), no further analyses into this interaction were undertaken.

Figure 3 presents the mean number of reversals for the 18 observers run in the three instructional conditions as a function of the four weekly sessions. The data have been collapsed across blocks and intervals for clarity of presentation. As in the previous

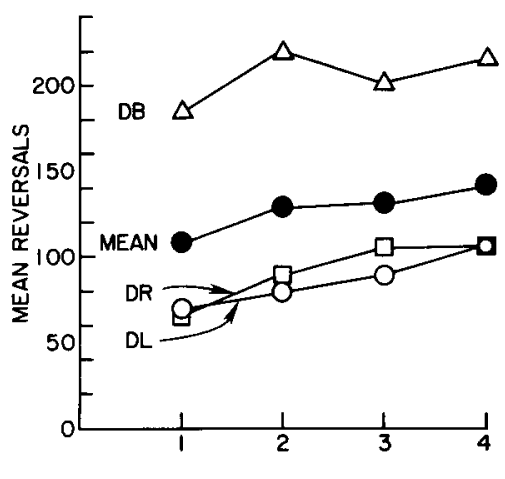

SUCCESSIVE WEEKLY SESSIONS

Figure 3. Mean reversals of the rotating Necker cube(s) reported in the three viewing conditions as a function of four weekly sessions. The curve labeled "MEAN" presents the data collapsed across the three viewing conditions. two figures, the main effect of conditions was highly significant $[F(2,15)=11.53, p<.01]$, with more reversals reported in the $\mathrm{DB}$ condition than in either the DL or DR condition ( $\mathrm{p}<.01$ by a Tukey a test). The main effect of sessions was also significant $[F(3,45)=3.83, p<.05]$. This results from the consistent increase in reversals over successive sessions. The interaction of conditions $\times$ sessions was not significant $(F<1.0)$.

\section{DISCUSSION}

As detailed in the introduction, the present research was aimed at evaluating the contribution made to perceived reversals of a rotating Necker cube by both a passive, fatigue-like process, on the one hand, and a learning/decisional process, on the other. For ease of discussion, then, the results will be treated in terms of the particular process(es) they are thought to reveal most directly.

\section{Evidence for Satiation}

Reversals over successive intervals. A common finding in the reversible-figure literature has been an increase in reported reversals over a viewing period, frequently with an asymptotic reversal rate after 1-2 min of viewing (cf. Price, 1969b; Vickers, 1972). This has been interpreted in terms of an alternation between cortical organizations which fatigue more and more rapidly over a viewing interval until a relatively constant rate of fatigue and recovery is attained. In the present work, the results in Figure 1 are generally supportive of this interpretation. For all instructional conditions, the number of reversals increased significantly within the 2 -min viewing block. Somewhat surprisingly, there was little evidence for a negatively accelerating function, which has frequently been reported for other reversible figures (cf. Babich \& Standing, 1981; Vickers, 1972). It was unknown whether this represented a qualitative difference between the rotating Necker cube and other reversible figures or whether the 2 -min viewing interval was not sufficiently long to reveal the asymptotic function. To assess the feasibility of the latter proposal, an additional group of 21 observers were run in the DL, DR, and DB conditions (seven subjects each) with 3-min viewing blocks rather than the 2min blocks employed with the previous subjects. Specifically, the observers received four 3-min blocks separated by 2 -min rest periods. A 3 (DL, DR, DB conditions) $\times 6$ (30-sec intervals) $\times 4$ (3-min blocks) ANOVA was performed on the results. The identical pattern of effects reported in Results above was obtained: The main effects of conditions, intervals, and blocks were again significant ( $p<.01$ in all cases). This represents a strong replication of the findings just reported. Figure 4 presents the mean number of reversals for the three instructional conditions as a 


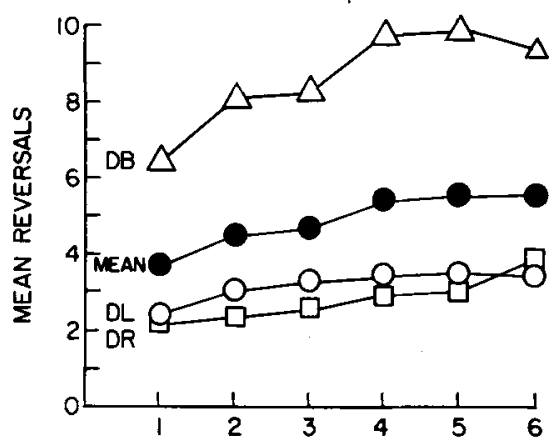

SUCCESSIVE 30-SEC INTERVALS IN THREE-MIN VIEWING PERIODS

Figure 4. Mean reversals of the rotating Necker cube(s) reported in the three viewing conditions as a function of the 30-sec intervals comprising the $3-\mathrm{min}$ viewing periods. The curve labeled "MEAN" presents the data collapsed across the three viewing conditions.

function of the six 30-sec intervals comprising the 3-min viewing blocks. Compared with Figure 1, there is somewhat stronger evidence for an asymptotic function. A Tukey a test was used to probe the conditions main effect, and no significant difference in reversals occurred between the last three intervals, indicating a general leveling out of reversals within the 3-min viewing period. Figure 5 presents the mean reversals for the three instructional conditions as a function of the successive 3-min blocks. The results are very similar to those presented in Figure 2 for the 2-min blocks.

It should be noted that a fatigue process is not the only possible explanation for an increase in reported reversals or for an eventual asymptoting of reversals during a viewing interval. As others have pointed out (cf. Ammons, 1954; Girgus et al., 1977; Sadler \& Mefferd, 1970; Thetford, 1963), a learning interpretation may also be proposed for such a pattern of results. However, the standard learning accounts of such an effect are either inadequate or insufficiently developed. If the increase in reversal rate during a 2 -min viewing period were attributable to learning, the learning curve might become negatively accelerated as each subject approached his/her maximum reversal rate. However, this simple learning explanation does not account for the data of the present experiment, in which the asymptote, which was approached in each viewing period, increased from one 2-min block to the next. What is needed is an explanation that would allow the learning curve to asymptote within each 2-min block, even though the maximum rate of reversals was not being approached.

One possible explanation depends on the constructs of learning and reactive inhibition (Ammons et al., 1959). Learning would account for the increase in reversals over time, and the buildup of reactive inhibition would explain why the rate of increase slows and eventually asymptotes. This account, however, leads to another prediction based on the fact that reactive inhibition is generally assumed to dissipate with time. When rest intervals separate viewing periods, the rate of reversal should increase from the end of one session to the beginning of the next. Unfortunately, results obtained in the present experiment, as well as in previous studies (e.g., Spitz \& Lipman, 1962), are in exactly the opposite direction. Reversal rates decrease from the end of one viewing session to the beginning of the next one. These findings cast serious doubt on the learning/reactive inhibition hypothesis, and, to the best of our knowledge, no other learning hypothesis has been developed in sufficient detail to provide a rigorous account of these details.

Reversals over instructional conditions. Of particular interest in the present work was the number of reversals reported in the $\mathrm{DB}$ condition relative to the number reported when only a single cube was attended to (DL and DR). If a reported reversal required a change in "attention" or a new "decision" by the observer, the reporting of reversals for two cubes simultaneously would require a spreading of the attentional/decisional process(es) over two concurrent stimuli. Unless one is willing to accept multiple concurrent decision processes or unlimited attentional mechanisms, a condition that requires the monitoring of two cubes (i.e., DB) would seem to require fewer reported reversals per cube than a condition in which only a single cube is monitored. No such effect was evidenced. As noted above, the number of reversals reported for the left cube in the DB condition or for the right cube in the $\mathrm{DB}$ condition was never less than the number of reversals for the

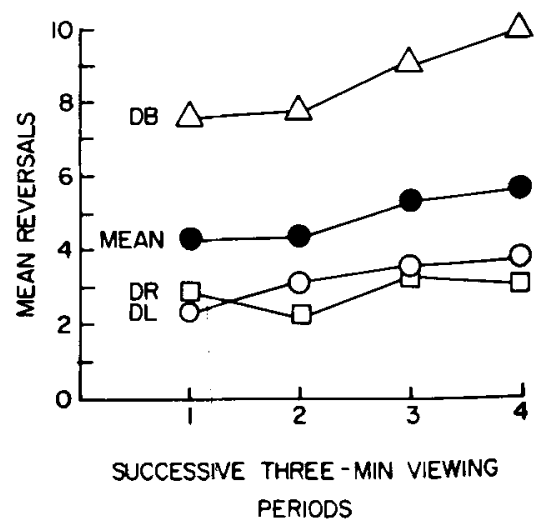

Figure 5. Mean reversals of the rotating Necker cube(s) reported in the three vlewing conditions as a function of the fow $3-\mathrm{min}$ viewing periods comprising the experimental sesolon. The cuirve labeled "MEAN" presents the data collapsed acrose the three viewing conditions. 
single cube monitored in the DL or the DR condition, respectively. Furthermore, the 21 observers run in the control condition with 3-min blocks exhibited the same pattern of results. The mean number of reversals reported in the DL condition (3.21) did not differ significantly from the number of reversals reported for the left cube in the DB condition (3.95) $[t(12)=.39, p>.05]$, and the number of reversals reported in the DR condition (2.86) did not differ significantly from the number of reversals reported for the right cube in the DB condition (4.73) $[\mathrm{t}(12)=$ $1.99, \mathrm{p}>.05]$.

This demonstration of the basic equivalence in the number of reversals reported for a cube when it is monitored singly or with a second cube in the other visual field is thought to be particularly consistent with the satiation model which attributes reversals to the fatiguing of cortical organizations (or channels). In this view, the DL condition describes the alternation between organizations in the right hemisphere, the DR condition describes the alternation between organizations in the left hemisphere and the DB condition describes the simultaneous alternation between organizations in both hemispheres. That is, number of reversals in $\mathrm{DB}=$ number of reversals in DL + number of reversals in DR. Following Long and Toppino's (1981) argument, this result would appear to cause difficulty for the strict attentional/decisional model of reversible figures such as that of Gregory (1974) or Rock (1975). ${ }^{3}$

It is realized that this "support" for the satiation model rests on the failure to reject the null hypothesis, the interpretation of which requires an exercise of caution. However, in the present study, the same result has been obtained for both the 2-min and the 3-min viewing conditions. In addition, there is a tendency for more reversals to be reported for each of the cubes in the DB condition than for the cube in either the DL or DR condition. Hence, it is highly unlikely that an increase in the power of the analysis by the addition of more observers would result in significantly fewer reversals for the cubes in the DB condition than for the cube in the DL or DR conditions, which is predicted by the limited-capacity decision model.

There is an alternate interpretation for the equivalent reversal rates of the individual cubes in the DB condition and the cubes in the DL and DR conditions that should be considered. Because there are two cubes in both the DL and the DR conditions (although the observer reports the reversals of only one), perhaps the observer is unable to restrict his/ her attentional or decisional processes to one cube and is therefore monitoring both cubes while explicitly reporting the reversals of only one of them. This interpretation of the present results would be entirely consistent with the decisional model described above by proposing that the limited decisional processes are shared between both cubes in the DL and DR conditions. It was for this reason that the two control conditions described in the Method section, SL and SR, in which only a single cube was presented, were included in this experiment. If there is no difference in the mean number of reversals in the four conditions (DL, DR, SL, and SR), then it can be assumed with some degree of confidence that in the DL and DR conditions observers were not dividing their limited resources across the two cubes in a way identical to that in the DB condition. An analysis of variance comparing the mean reversals in these four viewing conditions revealed no differences among them $[F(3,76)=1.53, p>.05]$. Hence, this interpretation, in terms of divided resources, is rejected.

\section{Evidence for Learning}

Effect of successive viewing blocks and sessions. The results shown in Figure 2 and Figure 5 appear to reflect a learning process within the experimental session. That is, there is a significant savings from each 2-min or 3-min block to the next. A passive fatigue process alone as the basis for reversals would be expected to produce nearly identical levels of reversals in each viewing period, provided that sufficient rest periods intervene to allow any buildup of fatigue to dissipate. The effect of blocks reported here is consistent with results of previous studies with other reversible figures that have also reported a significant learning effect (e.g., Adams, 1954; Ammons, 1954).

Of course, it is possible that the increase in reversals across blocks results from an incomplete recovery from fatigue of the neural channels in the intervening $2-\mathrm{min}$ rest period. This is thought to be unlikely for three reasons. First, extensive pilot work found that rest periods of 2 or 3 min did not differentially affect reversal rates. Second, each successive block showed the same pattern of increasing reversals across 30 -sec intervals, indicating that not only the starting level, but the entire pattern of reversals, was affected. Third, the results in Figure 3 show a clear increase in reversals for the 18 observers run over weekly intervals. This rather large effect, which is on the order of a $30 \%$ increase over a 4-week period, could hardly be attributed to incomplete recovery from neural fatigue. Hence, there is little doubt that learning plays a significant role in the number of reversals reported for a rotating Necker cube.

\section{CONCLUSIONS AND IMPLICATIONS}

It is proposed that the results of the present research support the claim made earlier that both neural fatigue and perceptual learning play important roles in perceived reversals of a rotating Necker cube. Moreover, such a two-process model of reversible figures can incorporate a wide range of empirical 
findings that otherwise present serious difficulty for those investigators favoring either satiation or learning as the single dominant process underlying perceptual reversals. For example, how is a strict learning/decisional model able to reconcile the finding that adaptation to an unambiguous version of a normally reversible figure results in a greater probability that an observer, upon being then presented with the standard reversible figure, will report the opposite version of the figure (e.g., Carlson, 1953; Harris, 1979, 1980; Hochberg, 1950; Virsu, 1975)? The satiation model, on the other hand, predicts this precise result because of the fatiguing of a particular channel by the prior adaptation. Conversely, how is a strict satiation model able to reconcile the finding that, if an observer is unfamiliar with the reversible character of a figure, there is a good chance that reversals of the figure will not be seen (Girgus et al., 1977)? A learning/decisional model has little difficulty with this result.

A particularly parsimonious way to integrate learning and fatigue effects into a single reasonable model may be available in the "quasi-neural process model" proposed by Palmer and Bucher (1981) in the context of ambiguous triangles (see, also, Harris, 1980 and Ginsberg, Note 1). As noted earlier, this model suggests that perceptual reversals represent alternations between neural systems that have been identified by extensive physiological and psychophysical research involving neural channels (see Braddick et al., 1978; Regan, 1982). Minor modifications of this model may permit the retention of the passive fatigue component within a neural channels framework, for which there is so much support from other research, while at the same time providing the greater flexibility necessary to incorporate learning and experiential effects.

A likely form for the nature of the modifications in the Palmer and Bucher model is suggested in a very old observation related to reversible figures: There is invariably a "preferred" percept or version of a reversible figure (e.g., Adams \& Haire, 1958; Carlson, 1953; Harris, 1980; Olson \& Orbach, 1966; Orbach et al., 1963; Price, 1969a, 1969b; Wieland \& Mefferd, 1966, 1967). Palmer and Bucher (1981) attribute this to "bias mechanisms" within the neural systems, resulting in greater activation of a particular system. To go a step further, might not this bias result from prior experience which serves, for example, to lower the threshold for a particular neural system? In addition, suppose that the total organization of activity across neural channels that results in "Percept A" of a Necker cube requires a degree of learning in order to be recognized as a distinct perceptual event. Similarly, the alternate organization of activity across channels that results in "Percept B" of a Necker cube also requires prior learning to establish its phenomenal identity. Such a model retains the contribution from neural channels while incorporating a Hebbian (1949) concept of a learning-based organization of neural components. Although current neurophysiology has replaced the molecular. building blocks of Hebb's original model with neural channels, the notion of a critical role played by learning in the interpretation or organization of the pattern of activity (output) of these channels may remain feasible. This conceptualization would appear entirely consistent with Regan's (1982) recent model of visual behavior which proposes that visual processing can be divided into two sequential parts: (1) "a stage of analysis in which much or most visual information passes through orthogonal sets of filters [channels]," and (2) "the second stage of visual processing [which] includes cognitive and learned information processing"' (pp. 418-419). In this conceptualization, the satiation-like results in the present work, as well as in extensive other research, result from the stimulus-dependent pattern of fatigue and recovery among the neural channels. The evidence of learning obtained in the present work, as well as in extensive other research, would reflect the need for the "establishment" via experience of each perceptual alternative among the myriad of channel activity. This, for example, could explain the Girgus et al. (1977) result concerning the importance of an observer's prior experience with a reversible figure. And, too, learning may also produce changes in the requirements (e.g., threshold, number of input sources, etc.) for the neural organizations underlying the perceptual alternatives once they have been established. In the present context, this follows from the fact that the increase in reversals over successive viewing periods is essentially unaffected by whether the cube is viewed alone or with a second cube. Hence, the learning that underlies the increase would appear to be taking place within the individual channels.

Obviously, such a model is somewhat vague and incomplete. Numerous empirical findings must be reconciled with its premises. For some, this is not very difficult. Consider, for example, the replicated finding that a change in the retinal position of a reversible figure results in a significant reduction in perceived reversals (Babich \& Standing, 1981; Spitz \& Lipman, 1962). This could be readily interpreted within the proposed model in terms of a corresponding change in the cortical channels involved, thereby incorporating less fatigued organizations in the percept with a consequently lower reversal rate of the figure. For other findings, however, the explanatory value of the model is somewhat less clear. What about individual differences in reversal rates which can be very large (see Sadler \& Mefferd, 1970; Vickers, 1972)? What about the role of attention, which also seems to play an important role in perceived reversals (see Palmer \& Bucher, 1981)? It is believed that, with relatively little difficulty, the above model can be 
modified to incorporate these and numerous other phenomena in the extensive reversible figures literature. However, because the present work has no direct evidence for these effects, and because any arguments would be based totally upon supposition, it was thought best to leave their explicit treatment to later research efforts.

\section{REFERENCE NOTE}

1. Ginsberg, A. P. Visual information processing based on spatial filters constrained by biological data (Report No. AMRL-TR78-129, Vols. 1 and 2). Wright-Patterson Air Force Base, Ohio: Aerospace Medical Research Laboratory, December 1978.

\section{REFERENCES}

Adams, P. A. The effect of past experience on the perspective reversal of a tridimensional figure. American Journal of Psychology, 1954, 67, 708-710.

Adams, P. A., \& Haire, M. Structural and conceptual factors in the perception of double-cube figures. American Journal of Psychology, 1958, 71, 548-556.

Ammons, R. B. Experiential factors in visual form perception: I. Review and formulation of problems. Journal of Genetic Psychology, 1954, 84, 3-25.

Ammons, R. B., Ulrich, P., \& Ammons, C. H. Voluntary control of perception of depth in a two-dimensional drawing. Proceedings of the Montana Academy of Sciences, 1959, 19, 160-168.

Atrmenve, F. Multistability in perception. Scientific American, $1971,225,62-71$.

Babich, S., \& Standing, L. Satiation effects with reversible figures. Perceptual and Motor Skills, 1981, 52, 203-210.

Beverley, K. I., \& Regan, D. Evidence for the existence of neural mechanisms selectively sensitive to the direction of motion in space. Journal of Physiology (London), 1973, 235, 17-29.

Blakemore, C., \& Campbell, F. W. On the existence of neurons in the human visual system selectively sensitive to the orientation and size of retinal images. Journal of Physiology (London), $1969,203,237-260$.

Blakemore, C., \& Nachmias, J. The orientation specificity of two visual after-effects. Journal of Physiology (London), 1971, 213, 157-174.

Blakemore, C., \& Sutron, P. Size adaptation: A new aftereffect. Science, 1969, 166, 245-247.

Braddick, O., Campbell, F. W., \& Atxinson, J. Channels in vision: Basic aspects. In R. Held, H. W. Leibowitz, \& H. L. Teber (Eds.), Handbook of Sensory Physiology (Vol. 8): Perception. Berlin: Springer, 1978.

Boring, E. G. Sensation and perception in the history of experimental psychology. New York: Appleton-Century-Crofts, 1942.

Brown, K. T. Rate of apparent change in a dynamic ambiguous figure as a function of observation time. American Journal of Psychology, 1955, 68, 358-371.

Campbell, F. W., \& Robson, J. G. Application of Fourier analysis to the visibility of gratings. Journal of Physiology (London), 1968, 197, 551-566.

Carlson, V. R. Satiation in a reversible figure. Journal of Experimental Psychology, 1953, 45, 442-448.

Chase, W., \& Smith, R. Spatial frequency channels tuned for depth and motion. Vision Research, 1981, 21, 621-625.

Cohen, L. Rate of apparent change of a Necker cube as a function of prior stimulation. American Journal of Psychology, $1959,72,327-344$.

Ellis, S. R., Wong, J. H., \& Stark, L. Absence of accommo- dation during perceptual reversal of Necker cubes. Vision Research, 1979, 19, 953-955.

Favreat, O. E. Persistence of simple and contingent motion aftereffects. Perception \& Psychophysics, 1979, 26, 187-194.

Flamm, L. E., \& Beroum, B. O. Reversible perspective figures and eye-movements. Perceptual and Motor Skills, 1977, 44, 1015-1019.

Frome, F., Harris, C. S., \& Levinson, J. Z. Extremely long lasting shifts in perception of size after adaptation to gratings. Bulletin of the Psychonomic Society, 1975, 6, 433. (Abstract)

Girous, J. J., Rock, I., \& Egatz, R. The effect of knowledge of reversibility on the reversibility of ambiguous figures. Perception \& Psychophysics, 1977, 22, 550-556.

Gregory, R. L. Choosing a paradigm for perception. In E. C. Carterette \& M. P. Friedman (Eds.), Handbook of perception (Vol. 1): Historical and philosophical roots of perception. New York: Academic Press, 1974.

Hansel, C. E. M., \& Mahmud, S. H. Comparable retention times for the negative colour afterimage and the McCollough effect. Vision Research, 1978, 18, 1601-1605.

Harkis, J. P. The Schroder staircase: A new perspective. Perception \& Psychophysics, 1979, 26, 312-318.

Harris, J. P. How does adaptation to disparity affect the perception of reversible figures. American Journal of Psychology, $1980,93,445-457$.

Heвв, D. O. Organization of behavior. New York: Wiley, 1949.

Hochbe RG, J. Figure-ground reversal as a function of visual satiation. Journal of Experimental Psychology, 1950, 40, 682-686.

Howard, I. P. An investigation of a satiation process in reversible perspective of revolving skeletal shapes. Quarterly Journal of Experimental Psychology, 1961, 13, 19-33.

KoHLen, W. Dynamics in psychology. New York: Grove Press, 1960.

Kohler, W., \& Wallach, H. Figural aftereffects, an investigation of visual processes. Proceedings of the American Philosophical Society, 1944, 88, 269-357.

Levinson, E., \& Seruler, R. A two-dimensional analysis of direction-specific adaptation. Vision Research, 1980, 20, 103-108.

Long, G. M., \& Toppino, T. C. Multiple representation of the same reversible figure: Implications for cognitive decisional interpretations. Perception, 1981, 10, 231-234.

Mafre I, L. Spatial frequency channels: Neural mechanisms. In R. Held, H. W. Weibowitz, \& H. L. Teuber (Eds.), Handbook of sensory physiology (Vol. 8): Perception. Berlin: Springer, 1978.

Mefferd, R. B., Jr., Wieland, B. A., Greenstein, D. G., \& LEPPMAN, P. K. Effects of pretraining and instructions on validity of perceptual reports by inexperienced observers. Perceptual and Motor Skills, 1968, 27, 1003-1006.

Olson, R., \& Orbach, J. Reversibility of the Necker cube: VIII. Parts of the figure contributing to the perception of reversals. Perceptual and Motor Skills, 1966, 22, 623-629.

Orbach, J., Ehrlich, D., \& Heath, H. A. Reversibility of the Necker cube: I. An examination of the concept of "satiation of orientation." Perceptual and Motor Skills, 1963, 17, 439-458.

Palmer, S. E., \& Bucher, N. M. Configural effects on perceived pointing of ambiguous triangles. Journal of Experimental Psychology: Human Perception and Performance, 1981, 7, 88-114.

Petersik, J. T., Beverley, K. I., \& Regan, D. Contrast sensitivity of the changing-size channel. Vision Research, 1981, 21, 829-832.

Pelton, L. H., \& Solley, C. M. Acceleration of reversals of a Necker cube. American Journal of Psychology, 1968, 81, 585-588.

Pheiffer, C. H., Eure, S. B., \& Hamilton, C. B. Reversible figures and eye-movements. American Journal of Psychology, $1956,69,452-455$.

Price, J. R. Effect of extended observation on reversible perspective duration. Psychonomic Science, 1969, 16, 75-76. (a) 
Price, J. R. Studies of reversible perspective: A methodological review. Behavior Research Methods \& Instrumentation, 1969, 1, 102-106. (b)

Pritchard, R. M. Visual illusions as stabilized retinal images. Quarterly Journal of Experimental Psychology, 1958, 10, 77-81.

REGAN, D. Visual information channeling in normal and disordered vision. Psychological Review, 1982, 89, 407-444.

REGAN, D., \& BEVERLEY, K. I. Illusory motion in depth: Aftereffects of adaptation to changing size. Vision Research, 1978, 18, 209-212.

Regan, D., \& Beverley, K. I. Binocular and monocular stimuli for motion in depth: Changing-disparity and changing-size feed the same motion-in-depth stage. Vision Research, 1979, 19, 1331-1342.

Regan, D., \& Cynader, M. Neurons in area 18 of cat visual cortex selectively sensitive to changing size: Nonlinear interactions between the response to two edges. Vision Research, 1979, 19 , 688-711.

Rock, I. An introduction to perception. New York: Macmillan, 1975.

Sadler, T. G., \& Mefferd, R. B. Fluctuations of perceptual organization and orientation. Perceptual and Motor Skills, $1970,31,739-749$.

Schume R, R., \& GANz, L. Independent stereoscopic channels for different extents of spatial pooling. Vision Research, 1979, 19, 1303-1314.

SpITz, H. H., \& Lipman, R. S. Some factors affecting Necker cube reversal rate. Perceptual and Motor Skills, 1962, 15, 611-625.

The Trond, P. E. Influence of massing and spacing on Necker cube reversals. Perceptual and Motor Skills, 1963, 16, 215-222.

Trtchener, E. B. Experimental psychology (Vol. 2): Quantitative. New York: Macmillan, 1905.

Vicholskovska, A. Illusions of reversible perspective. Psychological Review, 1906, 13, 276-290.

Vickens, D. A cyclic decision model of perceptual alternation. Perception, 1972, 1, 31-48.

Virsu, V. Determination of perspective reversals. Nature, 1975, 257, 786-787.

Wieland, B. A., \& Mefferd, R. B., Jr. Effects of orientation, inclination, and length of diagonal on reversal rate of Necker cube. Perceptual and Motor Skills, 1966, 23, 823-826.

Wieland, B. A., \& MefFerd, R. B., JR. Individual differences in Necker cube reversal rates and perspective dominance. Perceptual and Motor Skills, 1967, 24, 923-930.

Winer, B. J. Statistical principles in experimental design. New York: McGraw-Hill, 1971.

\section{NOTES}

1. Other explanations have, of course, been offered for the phenomenon of reversible figures. Some early investigators suggested that reversals resulted from changes in eye position (e.g.,
Necker, cited in Boring, 1942; Titchener, 1905). However, subsequent research demonstrated that reversals could take place without eye movements (e.g., Pritchard, 1958) and that eye movements may even result from the reversals rather than the other way around (e.g., Flamm \& Bergum, 1977; Pheiffer, Eure, \& Hamilton, 1956). Another early explanation involved accommodation changes which determined the "near" or "far" side of the Necker cube (e.g., Vicholskovska, 1906). Recently, Ellis, Wong, and Stark (1979) demonstrated that there was no relationship between accommodation and perceived reversal.

It is believed that the majority of other explanations can be classified into two large categories: satiation-based theories and theories based on learning and decision-making processes. Nevertheless, it is recognized that there are differences within these categories. In addition, the models proposed by some investigators tend to blur the distinction between the two categories. For example, the interesting "cyclic decision model" of Vickers (1972) is couched in decision terms and is even described in a decisional flowchart. Yet the details of the model involve a rather automatic set of processes that one might easily attempt to relate to the underlying neurophysiology. In other words, apparent differences in the models proposed to account for perceptual reversals may at times simply reflect the level of discourse favored by the particular investigator rather than qualitatively different processes.

2. These assumptions are based on the following considerations. First, according to decisional theories, the different interpretations of a reversible figure are seen successively because the related perceptual decisions are made serially. If these decisions could occur in parallel, simultaneous perceptions of both alternatives of a single cube would be possible. Second, any serial decision process seems limited in the number of decisions per unit of time although the practical extent of the limitation depends on the speed with which decisions can be made. Third, it is tecognized that one might assume the existence of multiple serial decision makers (one for each of an indefinite number of figures) that function in parallel with one another without interference. However, if one chooses a model with these unwieldy characteristics, the functional distinctions between decisional models and satiation models begin to blur.

3. As one of the reviewers pointed out, it is important for this argument against the decisional model that the two cubes in the $\mathrm{DB}$ condition not behave identically, reversing to the left or right in unison. If this were the case, it could plausibly be argued that a single decision is the basis for the perceived direction of rotation of both cubes. As Long and Toppino (1981) have noted, however, reports from observers indicate that reversals of either of the two cubes frequently occur independently of the behavior of the other cube. In fact, if an observer views four rotating cubes, some of the cubes may be seen rotating to the left, others to the right. Hence, a single decision does not appear to underlie the perceived direction of rotation in a multiple-cube condition.

(Manuscript received November 3, 1982; revision accepted for publication March 14, 1983.) 